\title{
Machine Learning Methods for Barret's and Dysplasia Classification from In Vivo Optical Coherence Tomography Images of Human Esophagus
}

\author{
Christos Photiou $^{1}$, George Plastiras ${ }^{1}$, Guillermo Tearney ${ }^{2}$, Costas Pitris ${ }^{1}$ \\ ${ }^{1}$ KIOS Research and Innovation Center of Excellence, Department of Electrical and Computer Engineering, \\ University of Cyprus, 75 Kallipoleos St, Nicosia, Cyprus, 1678 \\ ${ }^{2}$ Wellman Center for Photomedicine, Massachusetts General Hospital and Harvard Medical School, \\ 55 Fruit Street, Boston, MA 02114, USA.
}

\begin{abstract}
Machine learning methods comparison for esophagus tissue classification using in vivo OCT images. A neural network based approach discriminated better normal tissue from Barrett's Esophagus and dysplasia using various features with an accuracy of $87 \%$. (C) 2020 The Author(s)
\end{abstract}

\section{Introduction}

Endoscopic Optical Coherence Tomography (EOCT) systems can acquire cross-sectional images of the esophageal layers. Therefore, researchers can characterize the state of the esophageal tissue, discriminating between normal esophagus and Barret's Esophagus (BE) with or without dysplasia [1,2]. Computer-aided diagnosis can provide esophagus tissue characterization and classification between malignant and benign regions from OCT images [3,4]. In this study, we classify different regions of esophageal OCT images, utilizing a fully automated algorithm for image segmentation and feature extraction. Several machine learning classifiers were evaluated for accuracy and capability to differentiate normal from abnormal tissues and BE vs. dysplasia from in vivo acquired data.

\section{Methods}

\section{A. Image and Data Processing}

The data in this study collected with a swept source EOCT system with a center wavelength of $1300 \mathrm{~nm}$, an axial resolution of $10 \mu \mathrm{m}$ and an A-Scan rate of $40 \mathrm{kHz}$. In vivo data was collected from healthy volunteers and patients with esophageal disease enrolled in a study at Massachusetts General Hospital, approved by the Partner's Internal Review Board (IRB). Normal, BE or dysplastic tissue regions were annotated on the OCT images by an expert and tissue epithelium was automatically segmented and was used to extract image features.

\section{B. Feature Extraction}

The classification of the esophageal tissue was performed using various features extracted from the epithelial portion of the OCT images. These features included first order intensity statistics from the upper and lower part of the tissue epithelium and sliding standard deviation of every statistic resulting in a total of 36 intensity-based statistics for every A-Scan. Also group velocity dispersion (GVD) calculated using the image speckle technique that is applicable to in vivo imaging beginning from the top surface of the epithelium to a depth of $\sim 0.5 \mathrm{~mm}$ [5]. Finally, the average scatter size for each AScan of the epithelial layer was estimated using the bandwidth of the correlation of the derivative (COD) method. The $\mathrm{COD}$ is a new spectroscopic metric that can be used to calculate the average scatterer size using the first derivative of the spectrum followed by its autocorrelation. [6]

\section{Feature Selection and Classification}

In order to select the features that result in the best classification results, feature selection and optimization was performed. This process included utilization of a paired t-test and Multivariate Analysis of Variance (MANOVA). For the classification, five classifiers were, initially, evaluated: Discriminant Analysis (DA), Naïve-Bayes (NB), Decision Trees (DT), $k$-nearest neighbor (KNN) and Ensemble of Decision Trees (EDT). The performance of each classifier model was verified using leave-one-out cross-validation and was, subsequently, applied to entire images and volumes. Subsequently, using Python and Keras, different neural networks (NN) were constructed, varying the number of hidden layers and neurons, and each one evaluated on the same dataset. Each neighborhood of the epithelium was classified first as normal vs. abnormal and, subsequently, the abnormal areas were classified as BE vs. dysplasia. [7-10] 


\section{Results and discussion}

Figure 1 shows an example of an in vivo OCT image of the esophagus. In real-time, the image is displayed in the standard Cartesian coordinates. For processing purposes, the image was retained in polar coordinates (Fig.1A) and the image regions were annotated as normal, BE and dysplasia (Fig. 1A, blue boxes). The epithelium was automatically segmented thus, identifying the top and bottom boarders of the epithelium (Fig. 1A, red and green lines). The segmented regions (Fig.1B) were used in the machine learning process.
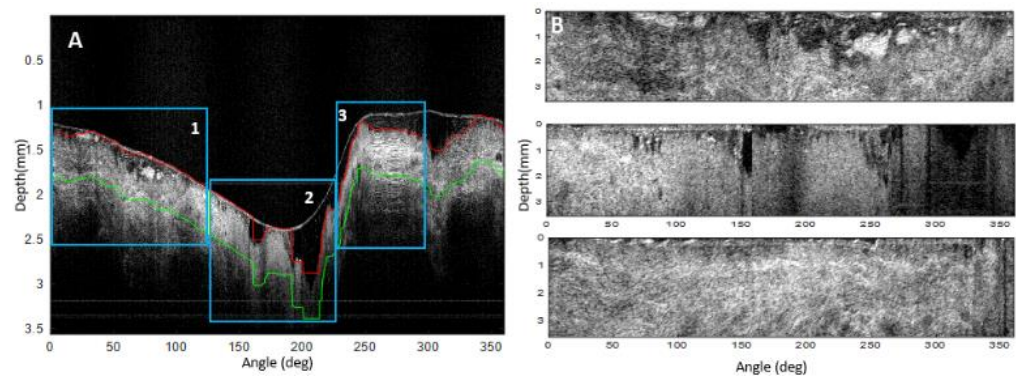

Fig. 1. (A)In vivo image in polar coordinates with the red and green lines indicating the top and bottom borders of the automatically segmented epithelial region. The blue boxes indicate annotated dysplastic (1), BE (2) and Normal (3) regions. (C) Zoomed regions corresponding (from top to bottom) to the dysplastic, BE and normal annotated areas respectively.

Table 1 shows the performance of all models. The performance of each classification scheme was verified using a leaveone image-out-cross validation approach for the same features selected based on t-testing and MANOVA (p-value <0.05). Mean, variance and median, of both the upper and lower parts of the segmented epithelial regions, along with the maximum of the lower area and the GVD appeared to be the most significant features. Normal vs. abnormal discrimination was more challenging to perform with the accuracy ranging from 63 to $73 \%$, sensitivity from 25 to $64 \%$ and specificity from 57 to $80 \%$. The neural network approach had the best accuracy (73\%), 64\% sensitivity and $80 \%$ specificity. For Barrett's vs. dysplasia, the classifiers resulted in accuracy values ranging from 60 to $87 \%$, sensitivity from 45 to $93 \%$ and specificity from 60 to $91 \%$. Again, the neural network classifier resulted in the best performance with an accuracy, sensitivity and specificity of $87 \%, 93 \%$ and $60 \%$ respectively. The results show that this automated algorithm could be developed to perform in vivo OCT esophageal tissue segmentation and classification. Using machine learning with a neural network, the algorithm can distinguish BE from dysplasia with an accuracy of $87 \%$, sensitivity of $93 \%$ and specificity of $60 \%$. These results are very promising and improve on the current state of the art but further evaluation is needed for effective computer aided diagnosis with OCT images from many patients.

Table 1. Normal vs Abnormal (left) and BE vs Dysplasia (right) classification using leave-one image out-cross-validation.

\begin{tabular}{|c|c|c|c|c|c|c|c|c|c|c|c|}
\hline N vs A & $\begin{array}{l}\text { Sensitivity } \\
{[\%]}\end{array}$ & $\begin{array}{l}\text { Specificity } \\
{[\%]}\end{array}$ & $\begin{array}{l}\text { Accuracy } \\
{[\%]}\end{array}$ & AUC & G-mean & B vs D & $\begin{array}{l}\text { Sensitivity } \\
{[\%]}\end{array}$ & $\begin{array}{l}\text { Specificity } \\
{[\%]}\end{array}$ & $\begin{array}{l}\text { Accuracy } \\
{[\%]}\end{array}$ & \multicolumn{2}{|c|}{ AUC G-mean } \\
\hline LDA & 29 & 62 & 67 & 0.66 & 0.42 & LDA & 80 & 91 & 74 & 0.57 & 0.85 \\
\hline k-NN & 31 & 57 & 64 & 0.63 & 0.41 & k-NN & 67 & 82 & 71 & 0.57 & 0.74 \\
\hline NB & 33 & 69 & 68 & 0.68 & 0.48 & NB & 45 & 71 & 60 & 0.63 & 0.56 \\
\hline EDT & 25 & 63 & 70 & 0.69 & 0.40 & EDT & 69 & 87 & 75 & 0.60 & 0.77 \\
\hline DT & 36 & 58 & 63 & 0.61 & 0.46 & DT & 57 & 77 & 69 & 0.59 & 0.66 \\
\hline NN & 64 & 80 & 73 & 0.76 & 0.72 & $\mathbf{N N}$ & 93 & 60 & 87 & 0.66 & 0.75 \\
\hline
\end{tabular}

\section{References}

1. G.Isenberg, et al, "Accuracy of EOCT in the detection of dysplasia in BE," Gast. Endos. Volume 62, 825-831, (2005).

2. Evans, et al. "OCT to identify intramucosal carcinoma and high-grade dysplasia in Barrett's esophagus," vol. 4, 38-43, (2006).

3. Qi,et al, "Image analysis for classification of dysplasia in Barrett's esophagus using EOCT," Biomed. Opt. Express 1, 825-847, (2010).

4. J. Ughi, et al, "Automated segmentation and characterization of esophageal wall in vivo by tethered capsule OCT, ” Biom.Op.Ex, 409-419, (2016)

5. C.Photiou, et al, "Using speckle to measure tissue dispersion in optical coherence tomography, ” Biomed. Opt. Express 8, 2528-2535 (2017).

6. Kassinopoulos, M., et al, "Correlation of the derivative as a robust estimator of scatterer size in OCT," Biomed. Opt. Express 8, 1598-1606 (2017).

7. Mika et al., "Fisher discriminant analysis with kernels," in Neural networks for signal processing society workshop., pp. 41-48, Ieee, (1999).

8. J.Quinlan, "Induction of decision trees," Machine learning, vol. 1, no. 1, pp. 81-106, (1986).

9. D. Opitz and R. Maclin, "Popular ensemble methods: An empirical study," Jour. of AI res vol. 11, pp. 169-198, (1999).

10. J. Erickson, et al, "Machine learning for medical imaging," Radiographics. 37(2): 505-515, (2017). 